TIPA. Travaux interdisciplinaires sur la parole et le langage

$31 \mid 2015$

L'impact du contact entre les langues

\title{
L'interférence comme particularité du « français cassé » en Algérie
}

Interference as a Particularity of "Broken French" in Algeria

\section{Hadjira Medane}

\section{(2) OpenEdition}

1 Journals

Édition électronique

URL : http://journals.openedition.org/tipa/1394

DOI : $10.4000 /$ tipa.1394

ISSN : 2264-7082

Éditeur

Laboratoire Parole et Langage

Référence électronique

Hadjira Medane, «L'interférence comme particularité du «français cassé » en Algérie », TIPA. Travaux interdisciplinaires sur la parole et le langage [En ligne], 31 | 2015, mis en ligne le 22 décembre 2015, consulté le 25 mai 2020. URL : http://journals.openedition.org/tipa/1394 ; DOI : https://doi.org/ 10.4000/tipa. 1394

Ce document a été généré automatiquement le 25 mai 2020.

La revue TIPA. Travaux interdisciplinaires sur la parole et le langage est mise à disposition selon les termes de la licence Creative Commons Attribution - Pas d'Utilisation Commerciale - Pas de Modification 4.0 International. 


\section{L'interférence comme particularité du « français cassé » en Algérie}

Interference as a Particularity of "Broken French" in Algeria

Hadjira Medane

\section{0 . Introduction}

1 La diversité linguistique en Algérie a fait l'objet de plusieurs recherches. Les faits sociolinguistiques qui en résultent déterminent le degré de contact et de brassage des langues en Algérie. Les langues présentes sur ce territoire, et qui coexistent les unes avec les autres, ne partagent pas la même importance ni le même prestige. La coexistence entre deux ou plusieurs langues implique qu'elles sont utilisées en fonction des situations, des régions et même des interlocuteurs par les membres de la société pour remplir diverses fonctions.

2 Le locuteur algérien exploite toutes les ressources langagières disponibles dans son paysage linguistique, ce qui a favorisé la créativité du sujet parlant algérien en français. Les pratiques langagières du français par les locuteurs algériens sont en perpétuelle évolution. Cette innovation "[...] se particularise dans sa forme et dans son contenu par un net décalage par rapport à la norme exogène du français standard »(Derradji, 1999 : 71).

3 En partant du constat de la complexité liée à la cohabitation des langues en Algérie, aux valeurs symboliques que ces langues véhiculent et de l'usage particulier du français dans ce pays, nous nous intéressons à ce que les Algériens appellent le «français cassé ». C'est un phénomène résultant des contacts entre le français et les langues ou les variétés de langues, y compris leurs représentations, qui se présentent dans le paysage linguistique algérien. C'est une réalisation fautive de la langue française liée, nous semble-t-il, à l'ensemble des images et des représentations de cette langue. Il s'agit donc d'un usage particulier du français. Cette pratique langagière constitue la particularité du français parlé en Algérie. Il s'agit d'un usage de la langue française 
dans lequel plusieurs phénomènes sont présents: interférence, calque, néologie, emprunt, etc.

4 La présente étude constitue une partie d'une enquête sur les particularités du «français cassé » utilisé en Algérie. Notre travail vise la description et l'analyse des manifestations de ce phénomène. Nous proposons une approche qualitative à travers l'analyse des productions de 20 jeunes algériens. En nous basant sur le principe de l'analyse contrastive, nous allons voir comment l'interférence constitue une des particularités de cet usage. Cette étude permettra également de voir si les interférences linguistiques constituant l'usage du français dit "cassé » sont liées à des facteurs extralinguistiques et /ou à des représentations particulières.

\section{Le «français cassé » d'un point de vue sociolinguistique}

5 Le multilinguisme en Algérie s'organise autour de quatre langues présentes sur le marché linguistique. Il se compose essentiellement de l'arabe algérien (qui est luimême divisé en plusieurs variétés régionales), du berbère et de l'arabe classique ou conventionnel (pour l'usage de l'officialité). A tout cela s'ajoute la langue française (première langue étrangère du pays).

6 La société algérienne est donc bien une société bilingue dans la mesure où les deux langues, l'arabe et le français, sont constamment utilisées. Ce bilinguisme, imposé par les circonstances de l'histoire, est souvent associé au «bilinguisme scolaire». Taleb Ibrahimi considère que ce dernier concerne le "contact [entre l'arabe et le français], langues écrites, de culture et de civilisation différentes, bilinguisme renforcé par l'adoption de ces deux langues comme langue d'enseignement dans le système » (1997: 50).

7 Etant scolarisé depuis son jeune âge dans ces deux langues, le jeune algérien doit après dix ans de scolarisation être un bilingue maîtrisant parfaitement l'arabe et le français. La réalité est néanmoins toute autre. Taleb Ibrahimi ajoute un peu plus loin : "L'école algérienne ne produit pas de bilingues, mais plutôt des semilingues qui ne dominent vraiment aucune des deux langues » (1997: 50). Cela remet par conséquent en question la politique du système éducatif.

La sociolinguiste propose, de ce fait, de distinguer

[...]d'une part, le bilinguisme institutionnalisé dans le système éducatif depuis 1962 [...], dans l'appareil administratif et économique de l'Etat au sein duquel les deux langues se « partagent » les domaines - à l'arabe les secteurs de légitimité et de souveraineté nationales, au français les secteurs technologiques et économiques [...] et, d'autre part, le bilinguisme dans la réalité des pratiques des locuteurs algériens, dans la réalité de la communication, dans laquelle le français a laissé son empreinte non seulement dans les différents dialectes, mais a marqué aussi certains usages de la variété standard et enfin, participe d'une compétence bilinguale [...]. (Taleb Ibrahimi, $1997: 52$ )

Le code switching, l'emprunt, la néologie à base française et d'autres phénomènes qui résultent de l'interpénétration des langues sont autant de phénomènes langagiers qui témoignent de la bilingualité du locuteur algérien: «Les sujets parlants algériens 
développent en fait un langage hybride nourri à partir d'un brassage de langues et de cultures " (Bahloul, 2009: 257)

Le bilinguisme a constitué, de ce point de vue, l'objet de plusieurs études que ce soit à travers des réflexions théoriques ou même, et surtout, pratiques. Le rapport conflictuel des langues arabe et française ne dépend pas seulement des contextes d'usage de ces deux langues ou de leurs usagers mais de tout un univers de représentations qui accompagne ces deux langues dans la théorie et dans la pratique. Dans cet esprit, Taleb Ibrahimi ajoute :

Il n'est plus possible de voir dans ce bilinguisme uniquement l'utilisation alternative des deux langues mais aussi et surtout le rapport de concurrence et compétition qui s'instaure entre les deux langues, un rapport dialectique qui fait passer chacune d'elles -selon les interlocuteurs et la situation d'énonciation- du statut de la langue dominante à celui de la langue dominée et vice versa. (1997 : 53)

11 En Algérie, le français a le prestige de la langue de l'ouverture sur le monde occidental et la modernité. Il est largement étudié en raison de sa place dans ce pays et hors de celui-ci. Le français a aussi l'importance de la langue de l'enseignement supérieur scientifique et technique, contrairement à l'arabe qui ne peut pas assumer ces fonctions. Les fonctions attribuées au français font de ce dernier une langue de l'information scientifique et technologique, du développement ; c'est pour cela qu'il est considéré comme la langue des lettrés et de la couche sociale la plus favorisée. Cela montre que le français est doté d'un certain prestige qui a sûrement des influences sur les représentations et donc sur les pratiques langagières des Algériens. Le prestige lié à la langue française en tant que langue de l'élite et de la démarcation sociale a un impact sur son emploi.

12 L'envie d'utiliser le français, représenté positivement dans plusieurs situations, a donné naissance à un phénomène sociolinguistique qui apparaît sous forme d'une utilisation fautive. C'est ce que les locuteurs algériens appellent «le français cassé» ou "le français déformé » ou même "le français à l'algérienne ». "Ne tombez pas les mots " pour " n'insultez pas ", " couper la route " pour " traverser la rue ", « tu casses mon tête " pour " tu me casses la tête ",... sont autant d'exemples de ce phénomène.

13 Le « français cassé » est une forme d'usage du français qui ne dépend pas du milieu socioculturel du locuteur algérien. Les jeunes issus de familles cultivées et même ceux issus de parents analphabètes sont des usagers du " français cassé ».

Le «français cassé » est, par ailleurs, utilisé par les jeunes algériens sans être dépendant d'une compétence en français. Même ceux qui disent maîtriser la langue française déclarent être usagers $d u$ "français cassé ». Cela est expliqué par une habitude langagière d'usage marquant une spécificité du français parlé en Algérie. Il s'agit des formes purement françaises et/ou des formes abrégées ou hybrides caractérisant le comportement langagier du locuteur algérien par rapport à la langue française. Il est donc question de formules et de mots en français qui, en dépit de leur caractère fautif ou non, restent utilisés par les Algériens.

Ce phénomène ouvre plusieurs pistes de recherche comme la question de l'insécurité linguistique due à la distribution inégalitaire des fonctions de l'arabe et du français en Algérie; mais aussi du phénomène de l'hypercorrection qui se manifeste dans les milieux diglossiques. 


\section{Le français dans les pratiques langagières des Algériens : usages et interférences}

que soit le système et quelle que soit la spécificité de la situation de contact, le passage d'une langue à une autre se fait rarement sans interférences. Nous pouvons admettre que lors de l'usage de la langue française, l'Algérien, en tant que locuteur bilingue, subit l'influence du système de l'arabe. Du fait de l'influence de la langue maternelle, celui-ci subit souvent des interférences pour ce qui est de l'utilisation de la langue française.

Dans la préface du livre de Walter (1988: 9), «Le français dans tous les sens », Martinet décrit les rapports qu'entretient un locuteur avec sa langue maternelle dans les propos suivants : "Les rapports de l'homme avec sa langue sont d'une nature particulière. Il l'a apprise sans le vouloir. Elle s'est imposée à lui par simple contact avec son entourage. Elle a coïncidé pour lui avec la prise de conscience du monde dans lequel il vit ».

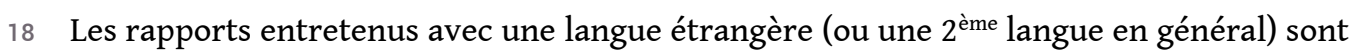
également particuliers mais aussi complexes car utiliser une langue étrangère n'est pas seulement se conformer à de nouvelles règles phonologiques, syntaxiques, morphologiques, lexicales, etc. - considérées comme des éléments structuraux -, mais aussi se conformer à des éléments non-structuraux qui peuvent être d'ordre socioculturel - tels que : l'âge, le milieu, le statut social - ou psychologique comme la loyauté envers la langue maternelle, l'aptitude individuelle, les attitudes personnelles ou stéréotypées, etc.

D'un point de vue structurel, le passage d'une langue à une autre devient plus complexe si les deux systèmes sont remarquablement différents. Les interférences produites dans le contexte algérien sont dues à la différence des systèmes du français et de l'arabe. Il s'agit de deux langues descendant de deux familles : « l'arabe descend de la famille Chamito-sémitique, le français de la famille Indo-européenne. Cela implique que les différences s'étendent aux aspects phonémique, morphologique, syntaxique, morphosyntaxique et lexical ». (Fesfes, 1994 : 9).

Dans ce sens, l'analyse de notre corpus se fera à travers la mise en évidence des différences d'organisation et de fonctionnement qui peuvent être à l'origine de la particularité de l'usage du « français cassé ».

En présence dans ce pays, le bilinguisme se fait de façon moins équilibrée entre les langues. Il a des effets sur les individus, sur les langues et même sur la société algérienne. Il s'agit à titre d'exemple d'une situation sociolinguistique dans laquelle la langue française laisse son empreinte dans les pratiques langagières.

21 Parmi les phénomènes liés au bilinguisme individuel (arabe/français) dans le contexte algérien, nous avons le phénomène de l'interférence. L'usage du « français cassé » par les jeunes interrogés a permis de dégager un nombre important d'interférences. Avant de passer à l'analyse des unités touchées par ce phénomène, nous allons - dans ce qui suit - présenter l'interférence linguistique et voir dans quelle mesure elle peut constituer une des particularités du « français cassé ». 


\section{L'interférence : présentation}

maternelle et de ses habitudes langagières. Selon Mackey (1976 : 414),

L'interférence est l'utilisation d'éléments d'une langue quand on parle ou écrit une autre langue. C'est une caractéristique du discours et non du code. Elle varie qualitativement et quantitativement de bilingue à bilingue et de temps en temps, elle varie aussi chez un même individu. Cela peut aller de la variation stylistique presque imperceptible au mélange des langues absolument évident.

La définition fournie par le Dictionnaire de linguistique et des sciences du langage (1994) montre que l'interférence se manifeste à des niveaux d'ordre phonologique, morphologique et syntaxique. On dit qu'il y a interférence "quand un sujet bilingue utilise dans une langue-cible L2, un trait phonétique, morphologique, lexical ou syntaxique caractéristique de la langue L1. ( (Kannas, 1994: 252).

Il est à noter que l'interférence est une pratique individuelle et involontaire. Elle est souvent considérée comme étant un écart par rapport à la norme. Dans ce sens, Hassan (1974:171) la définit comme "la violation inconsciente d'une norme d'une langue par l'influence des éléments d'une autre langue.». Abondant dans le même sens, Hamers considère l'interférence comme "une déviation par rapport aux normes des deux langues en contact » $(1994: 178)$.

Le phénomène de l'interférence se produit donc d'une langue à l'autre en présence de deux systèmes qui sont identiques sur des aspects et différents sur d'autres. Tout en utilisant la langue $B$, le locuteur se réfère aux traits de sa langue première ou langue maternelle, mais qui sont incompatibles avec celles de la langue B et choquent par conséquent le puriste.

Le locuteur algérien, comme tout utilisateur de la langue dans un pays multilingue, ne cesse de produire des interférences. Les différences linguistiques entre l'arabe et le français, sujet de notre recherche, entraînent forcément le problème d'interférence qui apparaît dans différents contextes.

L'interférence est souvent liée à une incompétence du locuteur bilingue dans la langue L2. Pour Hamers (1994: 178), "L'interférence se manifeste surtout chez des locuteurs qui ont une connaissance limitée de la langue qu'ils utilisent». Hagège la considère comme "un croisement involontaire entre deux langues. A grande échelle, l'interférence dénote l'acquisition incomplète d'une langue seconde. » (1996: 239).

\section{L'interférence comme caractéristique du « français cassé »}

\subsection{Présentation du corpus de la recherche}

Notre enquête a été faite dans deux villes algériennes: Tlemcen et Chlef. Nous avons demandé à 20 jeunes qui disent utiliser un «français cassé » de commenter un certain nombre d'images qui traitent des sujets ${ }^{1}$ en relation avec la jeunesse algérienne. Nous

TIPA. Travaux interdisciplinaires sur la parole et le langage, 31 | 2015 
avons procédé par enregistrement. A l'aide de cette technique d'investigation, nous visons à analyser les particularités du français dit « cassé » et voir en quoi il s'écarte de celui de l'Hexagone.

Après avoir écouté les enregistrements des enquêtés, nous avons recherché les particularités. Afin de pouvoir les regrouper en différents types, nous avons effectué plusieurs écoutes. D'abord nous avons tenté de les identifier puis nous les avons classées en vue d'une interprétation. La constitution et l'observation du corpus a été possible grâce à la transcription des productions enregistrées.

Après l'analyse de toutes les particularités, il apparaît que l'usage du français dit "cassé » est renvoyé, entre autres, au phénomène d'interférence. Notre corpus a permis de distinguer trois types d'interférence: phonétique, lexico-sémantique et syntaxique.

\subsection{Interférence phonétique}

La concomitance de deux codes chez le locuteur bilingue implique l'insertion d'une unité d'une langue dans une autre. Le phonème en tant que la plus petite unité constitutive du système de la langue constitue à son tour un lieu d'interférence. On parle d'interférence phonologique lorsqu'on arrive à identifier «[...] un phonème de la langue cible par l'intermédiaire d'un phonème de la langue maternelle; les deux phonèmes seront dès lors reconnus et réalisés comme étant absolument identiques » ((Dweik, $2000: 225)$, cité par Hasanat, $2007: 211-212$ ).

Les différences des systèmes phonologiques de l'arabe et du français entraînent la production d'un nombre important d'interférences dans le contexte algérien. Après avoir collecté, regroupé les erreurs relevant d'une interférence phonétique, nous avons élaboré le tableau ci-dessous :

\begin{tabular}{|c|c|c|}
\hline Erreur relevée (à l'oral) & Correction en API & Origine de l'erreur \\
\hline [fenoman] & {$[$ fenomen] } & \begin{tabular}{|l} 
Phonétique : $[\varepsilon] /$ [a] \\
Usage de la voyelle [a] à la place de $[\varepsilon]$
\end{tabular} \\
\hline \begin{tabular}{|l} 
[fim] \\
[siRtu] \\
[kJmin] \\
[siperjœe $]$ \\
[insigaRet]
\end{tabular} & \begin{tabular}{|l} 
[fym] \\
[syrtu] \\
[kJmyn] \\
[syperjœeR] \\
[ynsigaRet]
\end{tabular} & $\begin{array}{l}\text { Phonétique : }[\mathbf{y}] \text { / [i] } \\
\text { [y] confondu avec [i] }\end{array}$ \\
\hline [esurtulazœnes] & [esyRtulazœnes] & $\begin{array}{l}\text { Phonétique : }[\mathrm{y}] /[\mathrm{u}] \\
{[\mathrm{y}] \text { confondu avec }[\mathrm{u}]}\end{array}$ \\
\hline $\begin{array}{l}\text { [plekuRõ }] \\
\text { [obtone] }\end{array}$ & $\begin{array}{l}\text { [plykuRõ] } \\
\text { [obtəny] }\end{array}$ & $\begin{array}{l}\text { Phonétique : [y] / [e] } \\
\text { [y] tend vers [e] }\end{array}$ \\
\hline $\begin{array}{l}\text { [normalmo] } \\
\text { [malœrœzmo] }\end{array}$ & $\begin{array}{l}\text { [nכrmalmõ }] \\
{[\text { malœrœzmã }]}\end{array}$ & 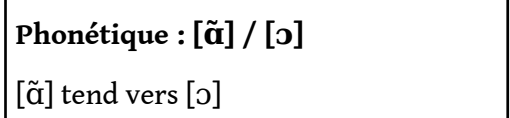 \\
\hline
\end{tabular}




\begin{tabular}{|c|c|c|}
\hline [donleklas] & [dõ̃leklas] & 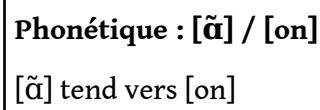 \\
\hline $\begin{array}{l}\text { [monas] } \\
\text { [obtoni] } \\
\text { [ãglot乏R] }\end{array}$ & $\begin{array}{l}\text { [mənas }] \\
\text { [obtəny }] \\
\text { [ãglətદR] }\end{array}$ & $\begin{array}{l}\text { Phonétique : [ə] / [o] } \\
\text { [ə] confondu avec [o] }\end{array}$ \\
\hline 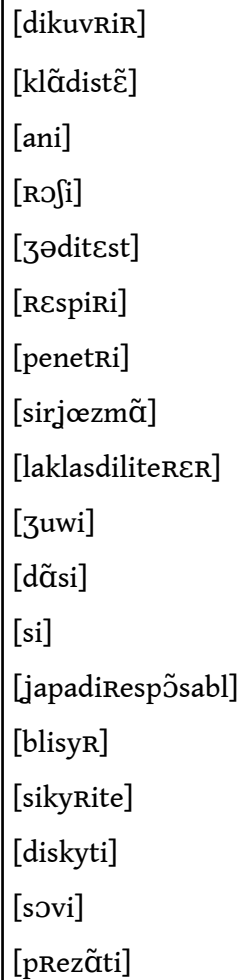 & 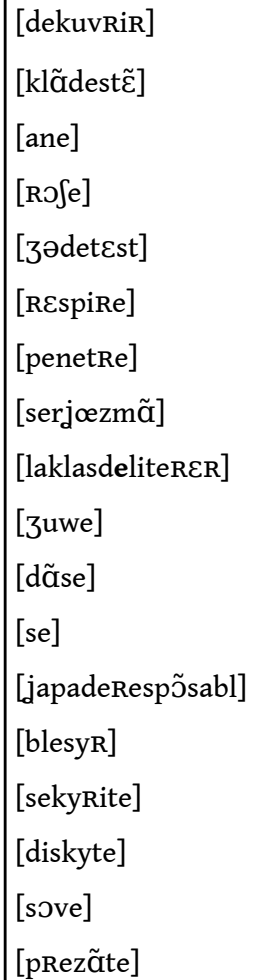 & $\begin{array}{l}\text { Phonétique : [e] / [i] } \\
\text { [e] confondu avec [i] }\end{array}$ \\
\hline [ãmblysdəsa] & [ãmplysdəsa] & $\begin{array}{l}\text { Phonétique : [p] / [b] } \\
\text { [p] confondu avec [b] }\end{array}$ \\
\hline
\end{tabular}

TABLEAU $\mathbf{N}^{\circ} \mathbf{1}$ : Erreurs relevant d'une interférence phonétique.

L'observation des données du tableau montre que les interférences du type vocalique sont omniprésentes. Il est à noter que nous n'avons rencontré qu'une seule erreur relevant d'interférences des systèmes consonantiques.

L'inventaire vocalique arabe est plus réduit que celui du français. Rappelons que le français possède seize voyelles (orales et nasales) tandis que l'arabe n'en possède que trois. Hasanat (2007) résume les caractéristiques vocaliques des systèmes arabe et français dans les tableaux ci-dessous :

\begin{tabular}{|c|c|}
\hline - Voyelles arabes: & - Voyelles françaises: \\
\hline 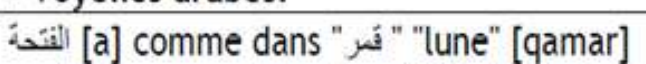 & [a] comme dans "chat" [ $[$ a]. \\
\hline 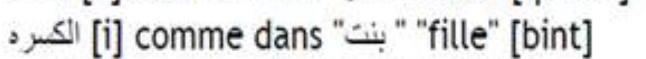 & [i] comme dans "fil" [fil]. \\
\hline [u] comme dans "بن " "café" [bun] & [u] comme dans "poupée" [pupe]. \\
\hline
\end{tabular}

TABLEAU N² 2 : Les voyelles communes entre l'arabe et le français (Hasanat, 2007 : 218). 


\begin{tabular}{|c|c|}
\hline Voyelles limitées à la langue arabe & Voyelles limitées à la langue française \\
\hline 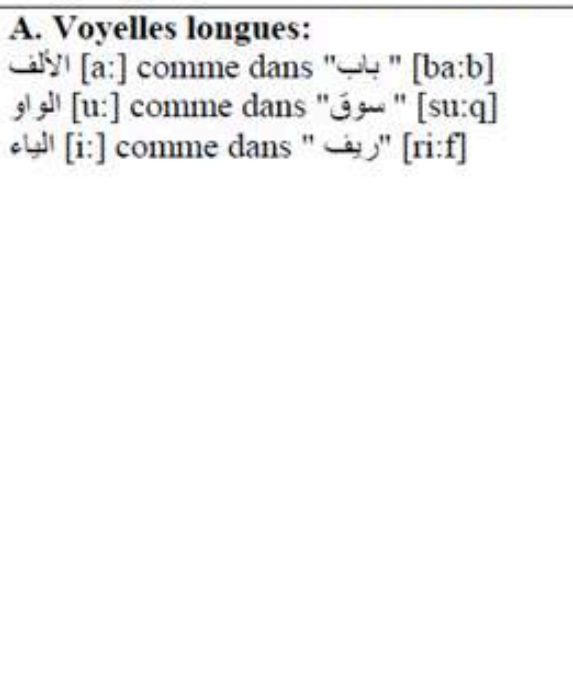 & 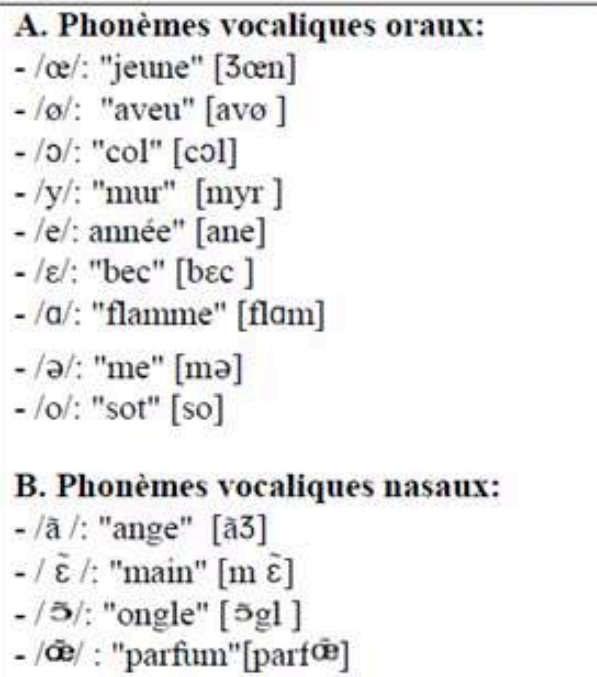 \\
\hline
\end{tabular}

TABLEAU $\mathbf{N}^{\circ} \mathbf{3}$ : Les voyelles limitées à la langue arabe et à la langue française (Hasanat, 2007 : 219).

Les différences relevées entre les deux systèmes vocaliques peuvent constituer des lieux privilégiés de réalisation de l'interférence chez nos enquêtés. Les facteurs de labialisation et de nasalisation caractérisent le système vocalique français. Ces facteurs sont inconnus au locuteur arabophone. L'absence des voyelles [y], [e], [ $\varepsilon],[ə]$ et des voyelles nasales du système vocalique arabe est à l'origine des interférences relevées de notre corpus. Ainsi :

- Le [y] (voyelle arrondie, très fermée en français) est soit prononcé [i], soit [u], soit [e]. C'est le cas par exemple de [siRtu] ou de [suRtu] au lieu de [syRtu] ou de [plekuRã ] au lieu de [plykuRã].

- Le $[\varepsilon]$ (voyelle non-arrondie, semi-ouverte, antérieure) est remplacé par un [a] tel qu'il apparait dans [fenoman] (au lieu de [fenomen]).

- Le [ə] (voyelle arrondie, fermée, antérieure) est souvent confondu avec [o] : comme dans la réalisation de [monas] au lieu de [mənas].

- Le [e] voyelle non arrondie, fermée et antérieure) est confondu avec [i] : c'est le cas de la majorité des interférences relevées (Voir tableau n ${ }^{\circ} 1$ ). Prenons à titre d'exemple [dekuvRiR] prononcé [dikuvRiR].

- Le [ã] (voyelle postérieure, non arrondie, ouverte, nasale) est substitué soit à un [0] : c'est le cas de [normalmã] prononcé [normalmo] ; soit elle est réalisée [on] comme dans [donleklas] au lieu de [dõ̃leklas].

Pour ce qui est du système consonantique, rappelons que celui de l'arabe est plus riche que celui du français. Le tableau suivant regroupe les phonèmes consonantiques communs à ces deux langues.

\begin{tabular}{|l|l|l|l|l|l|l|l|l|l|}
\hline Gr. & Ph. & Gr. & Ph. & Gr. & Ph. & Gr. & Ph. & Gr. & Ph. \\
\hline I & A & C & X & ش & S & $\dot{\varepsilon}$ & X (R) & ن & n \\
\hline C & B & د & D & ص & s $^{\mathrm{S}}$ & ف & F & $\circ$ & h \\
\hline
\end{tabular}




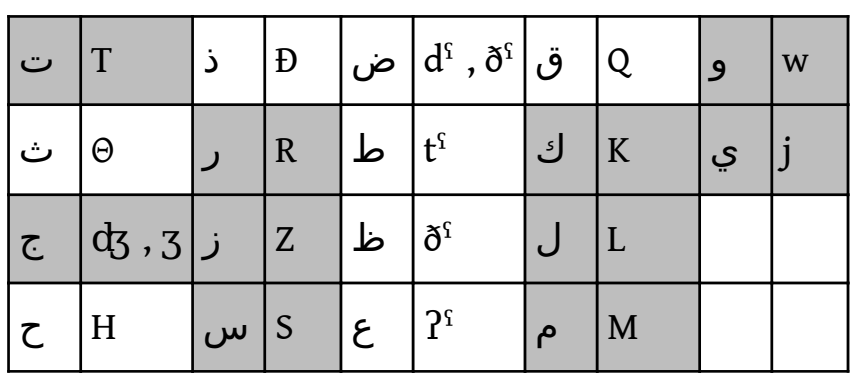

TABLEAU $\mathbf{N}^{\circ} \mathbf{4}$ : Les phonèmes consonantiques communs à l'arabe et au français.

Une comparaison des deux systèmes montre, dans la pratique, que l'Algérien éprouve des difficultés lors de la réalisation des phonèmes français [p], [v], [g] et [n], bien que ces phonèmes ne soient pas totalement inconnus de l'arabe algérien. Notre corpus a permis de montrer que le [p] (occlusif, bilabial, sourd) est souvent confondu avec [b] existant dans le consonantisme arabe comme dans l'exemple cité dans le tableau ${ }^{\circ} 1$ : [ãmply] est prononcé [ãmbly].

Les prononciations erronées caractéristiques d'interférences dans les productions des jeunes interrogés sont dues :

- aux habitudes articulatoires de l'arabe qui ne connaît pas de voyelles intermédiaires entre le [a], le [i] et le [u]. Bien qu'elle soit présente en arabe dialectal, la voyelle [e] (une voyelle intermédiaire) constitue aussi chez un bon nombre de locuteurs algériens une des difficultés articulatoires du français.

- à l'adaptation des voyelles et des consonnes française, qui n'existent pas en arabe, comme $[y],[e],[\varepsilon]$ et [ə] (pour les voyelles) et [p], [v], [g] et [n] (pour les consonnes). Cette adaptation se fait en prononçant des phonèmes de proximité articulatoire existant en arabe.

- à la dénasalisation des voyelles nasales en les réduisant à des voyelles simples proches d'un point de vue articulatoire : comme c'est le cas de [ã] prononcé [o] (ou [o]) ; ou en ajoutant à ces réductions un $[\mathrm{n}]$.

39 Ainsi, en utilisant le français, le locuteur algérien réduit le système phonétique (vocalique et consonantique) français aux phonèmes de l'arabe. A propos des voyelles, Maume précise que le locuteur arabophone «tend à éliminer les facteurs de labialisation et de nasalisation propres au français pour ne retenir que l'opposition voyelle antérieure/voyelle postérieure» (1973 : 104). Il est à signaler que, vu le nombre important des emprunts au français existant dans l'arabe dialectal, le locuteur algérien s'est familiarisé avec certains de ces phonèmes.

\subsection{Interférence sémantico-lexicale}

Ce type d'interférence concerne les unités lexicales que le locuteur bilingue introduit dans son discours. Ce transfert interlingual se fait lorsqu'un terme donné appartenant à la langue source fait intrusion dans la langue cible. Il ne faut pas confondre l'interférence lexicale avec l'emprunt. Une distinction qui s'avère pertinente est que la première est un fait individuel et inconscient tandis que le deuxième est social.

41 Les erreurs que nous avons relevées dans les productions des jeunes enregistrés sont dues aux interférences lexicales. A la suite des analyses que nous avons effectuées, nous avons trouvé des exemples d'erreurs créées par le recours à l'arabe. Nous pouvons regrouper ces erreurs dans les trois catégories représentées dans le tableau ci-dessous : 


\begin{tabular}{|c|c|c|}
\hline & Productions des enquêtés & Erreur relevée \\
\hline Catégorie -1- & [mat $\left.\int d ə s u d \varepsilon n\right]$ & [suden] \\
\hline Catégorie -2- & 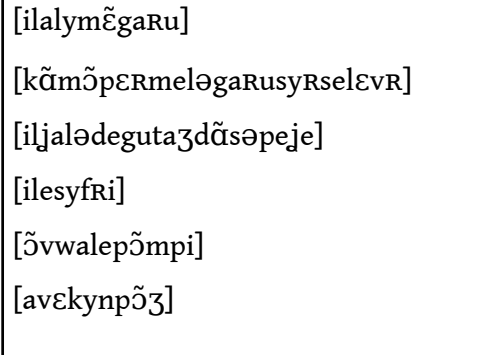 & $\begin{array}{l}\text { [gaRu] } \\
\text { [gaRu] } \\
\text { [degutaz] } \\
\text { [syfri] } \\
\text { [põmpi] } \\
\text { [põz] }\end{array}$ \\
\hline Catégorie -3- & 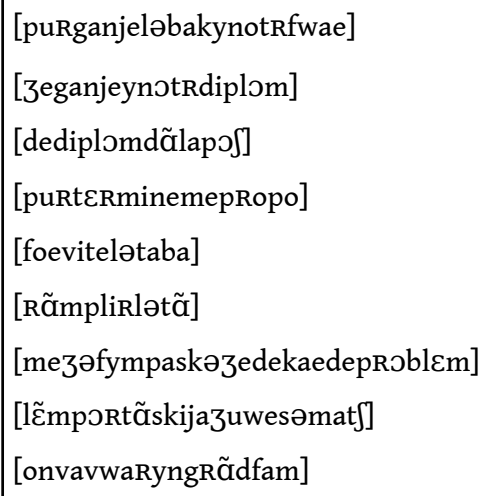 & 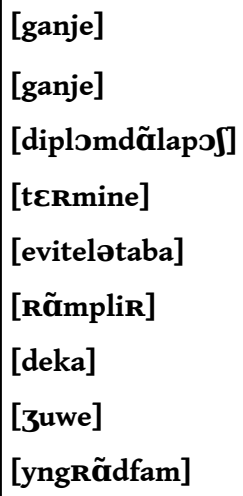 \\
\hline
\end{tabular}

TABLEAU N5 : Erreurs relevant d’une interférence lexicale. langue arabe. Les locuteurs adaptent une prononciation des mots tels qu'ils existent dans cette langue : [suden] (au lieu de [sudã]). (entème catégorie concerne le recours à des mots de l'arabe dialectal. Ces unités lexicales constituent soit des emprunts à différentes langues existant dans le parler algérien soit des néologismes à base française.

- Dans [ilalymẽgaRu] et [kốmõp๕RmeləgaRusyrselevr] les enquêtés utilisent le mot [gaRu] au lieu de « cigarette ». Cette interférence est due à l'usage d'un emprunt à l'espagnol utilisé dans l'arabe algérien (et même dans le tamazight)2. Il s'agit du mot espagnol: " cigarro ». L'intégration de ce terme a été faite par aphérèse c'est-à-dire par la suppression de la première partie du mot pour n'obtenir que « garro ».

- Dans [ilesyfRi] le jeune interrogé utilise le mot [syfri] au lieu de «il souffre». Il s'agit de l'usage de l'emprunt au français intégré dans le parler algérien [syfRi]. La même chose pour l'usage de [põmpi] dans [õvwalepõmpi] au lieu de «on voit le pompier ». L'interférence lexicale réside dans le fait d'utiliser l'emprunt intégré dans le discours.

- Pour ce qui est de l'énoncé [iljalədegutazdõsəpeje]le jeune interrogé a utilisé le néologisme [deguta3] utilisé dans le dialecte algérien au lieu de « l'ennui » ou « le dégoût ».

- Dans [avekynpõz] le locuteur utilise le mot [põz]. Il s'agit d'un recours à l'emprunt au français « éponge » intégré par aphérèse dans le dialecte algérien sous forme de [põza].

Dans ces exemples le terme emprunté retourne à la langue source mais de façon erronée. L'usage de ces termes constitue une des caractéristiques du «français cassé ». 
Le retour du terme emprunté, adapté et souvent intégré, à la langue prêteuse peut constituer un des éléments du changement linguistique.

Pour ce qui est de la troisième catégorie, elle concerne une importation du signifié du mot arabe puis son application au signifiant du mot français tout en le traduisant. C'est une des caractéristiques du calque. Ainsi :

- Dans [puRganjeləbakynotRfwae] et [3eganjeynotrdiplom] le jeune enregistré fait une interférence à l'arabe en utilisant le verbe "gagner 》 (équivalent de "ربح " [rabiHa] en arabe) au lieu de " avoir » (« avoir le bac » et « avoir un diplôme »).

- Dans [dediplomdãlapo]] le locuteur a commis une erreur due à une interférence lexicale pour dire "avoir un diplôme». Cette interférence est due au recours à l'expression de l'arabe dialectal « " chose ».

- Pour ce qui est de l'énoncé [puRterminemepropo], l'usage du mot [termine] dans ce contexte est dû à une interférence avec l'arabe. Le locuteur se réfère à l'expression : «إنهاء الحديث « انهاء " et utilise le mot [tERmine] équivalent de («pour conclure ») [liinHaialHadi] [inHa].

-L'usage de [foevitelətaba] au lieu de "il faut cesser de fumer» est un résultat d'une interférence lexico-sémantique. Le jeune interrogé fait recours au mot arabe "التدخين " [atadKhin] équivalent du «tabac » en français. le mot en arabe signifie à la fois «le tabac » et « le fait de fumer».

• En ce qui concerne [Rõ̃mpliRlətã], l'usage de [Rõ̃mpliR] (équivalent de "يعمر 》 [jÇamar] en arabe) est un indice d'interférence lexicale. Le jeune enregistré fait référence à l'expression . dans le sens de " combler le vide (ou [jÇamarlvid]) [jÇamarlwaqt] "يعمرالوقت"

-A propos de [3əfympaskəzedekaedeproblem] l'usager utilise le mot « cas » en se référant à son équivalent en arabe "حالة " [Hala]. Ce dernier est synonyme de "ظرف " [ðَarf]

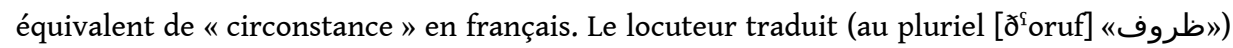
donc en français ce qu'on dirait en arabe [Çandið̊orufwmaJakil].

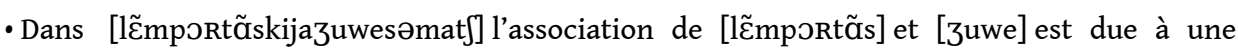
interférence avec l'arabe où l'équivalent du mot « importance » ( أهمية " أ [aHamija]) et celui du mot «rôle " (" دور " [dawr]) sont synonymes et peuvent être utilisés l'un à la place de l'autre. Par contre en français on n'associe [3uwe] qu'au mot «rôle». Le jeune interrogé utilise cette séquence pour indiquer " L'importance que le match avait ».

- L'usage de [gRãd] dans [onvavwaRyngRãdfam] est dû à une interférence lexicale. Le locuteur fait référence à sa langue maternelle où l'équivalent de "vieille femme » est [mrakbira] qu'il l'a traduit en français [gRõdfam].

\subsection{L'interférence syntaxique}

L'interférence syntaxique est le résultat d'une méconnaissance des règles de la langue cible. Tabouret-Keller affirme que dans le domaine de la grammaire "l'interférence linguistique déterminera des perturbations dans le jeu des règles de la syntaxe et dans celui du marquage fonctionnel spécifique de syntagmes » (2008:10). Il s'agit donc de l'introduction dans une langue du bilingue des unités ainsi que des combinaisons d'unités linguistiques provenant d'une autre langue. Cela se produit lorsque le bilingue transfère certaines caractéristiques grammaticales de sa langue $\mathrm{A}$ à la langue $\mathrm{B}$.

Les erreurs d'ordre syntaxique que nous avons relevées dans notre corpus constituent aussi des interférences syntaxiques. L'intrusion des traits syntaxiques de l'arabe dans le 
discours des jeunes interrogés caractérise le "français cassé ». Nous avons pu dans ce qui suit répertorier les erreurs interférentielles morphosyntaxiques qui se rapportent au genre des noms, à l'emploi de la négation, de la préposition et de l'article contracté, à l'emploi des pronoms personnels et des pronoms relatifs et enfin à la forme pronominale des verbes.

\subsubsection{Le genre des noms}

Une des caractéristiques du « français cassé » est la confusion du genre des noms. Il s'agit d'une interférence morphologique où le genre de l'équivalent du nom en arabe influence celui du français. Le tableau ci-dessous regroupe toutes les interférences du genre des noms relevées du discours des enquêtés.

\begin{tabular}{|c|c|}
\hline Production de l'enquêté & Erreur relevée \\
\hline [3eganjeynotRdiplom] & [ynotRdiplom] \\
\hline 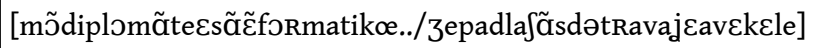 & {$[\varepsilon$ le $]$} \\
\hline [3eobtoneyntRvaj] & [yntRvaj] \\
\hline 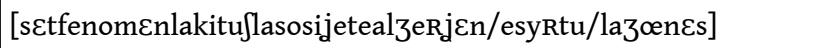 & [sctfenomen] \\
\hline [savediRsetmat [] & [sctmat $]]$ \\
\hline $\begin{array}{l}\text { [onvwaynaksidã/ynkamjõ] } \\
\text { [ynmotoafeynaksidõavekynkamjõ] } \\
\text { [vwaynaksidõdəvwtyR] }\end{array}$ & [ynaksidõ]/ [ynkamj̃̃] \\
\hline [ləmənysiynbatoavekleHaraga ] & [ynbato] \\
\hline [laprəmjદrpumõynpumõdynpersonkinəfympa] & [laprəmjદrpumõynpumõ] \\
\hline [eladøzjempumõ/dy/ynp\&Rsonfyme ] & [ladøzj̇єmpumõ] \\
\hline [lakulœRdəs\&tpumõilenwar] & [dəsctpumõilenwar] \\
\hline [3əvefRapeyntuR] & [yntuR] \\
\hline [folytesədRog] & [sədRog] \\
\hline [penetRiososijete] & [ososijete] \\
\hline [ləkupdymõd] & [ləkup] \\
\hline [lekotdivwaR] & [lekotdivwaR] \\
\hline [ləpєRsว̃aalze] & [ləpعRsõ] \\
\hline [eiljayndistãsavekləmєR] & [ləmعR] \\
\hline [seləfyme] & [ləfyme] \\
\hline
\end{tabular}




\begin{tabular}{|c|c|}
\hline [savədiRlafymile.../emכRtel ] & [lafymile.../] \\
\hline [presãtẽnaRm] & [ $\tilde{\varepsilon}$ naRm] \\
\hline [tykasmõtct] & [mõtct] \\
\hline
\end{tabular}

TABLEAU $\mathbf{N}^{\circ} \mathbf{6}$ : Le genre des noms comme source d'interférence.

En se référant à leur langue maternelle, les jeunes enquêtés ont utilisé l'article féminin pour des mots français masculins. C'est le cas de :

- [ynotRdiplom] : Le locuteur se réfère à l'équivalent du mot « un diplôme » en arabe. Il s'agit du mot "شهادة" [Sahada] qui est un mot féminin. L'usage du pronom [Ele] dans

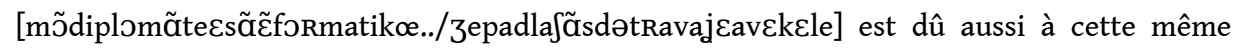
interférence.

•[yntRvaj] : Le jeune fait référence au mot féminin "خدمة " [xadma] de l'arabe dialectal équivalent de « un travail » en français.

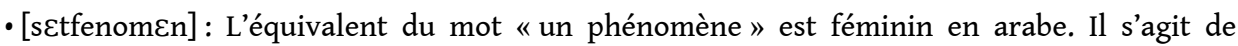

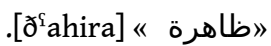

-[sctmatf] : Le locuteur fait référence à l'équivalent féminin du mot « un match » en arabe. Il s'agit de «مباراة" [mobarat]. Ce qui justifie l'emploi de [sct]

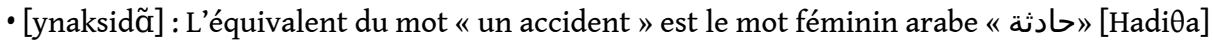

•[ynkamjõ] : Le jeune se réfère au mot féminin arabe "شاحنة [ [SaHina] équivalent de "un camion » en français.

•[ynbato] : La référence au mot féminin arabe «باخرة" [baxira] équivalent de «un bateau » est source de l'erreur dans ce mot.

- [laprəmj¿rpumכ̃ynpumõ] : L'équivalent du mot « un poumon » est féminin en arabe. Il s'agit de «رئة » [ria]. Ce qui justifie l'emploi de l'article «la». Cette interférence est la source de l'erreur dans [ladøzj¿̨mpumõ] et dans [dəsદtpumõilenwar].

• [yntuR] : Le mot "دورة [dawra] (équivalent de "un tour ») en arabe est féminin.

Un autre type d'erreurs interférentielles apparaît dans notre corpus quand nos enquêtés utilisent des articles masculins pour des mots féminins. Cela est dû au fait que le mot concerné a un équivalent masculin en arabe. Cela apparait notamment dans :

- [sədRog] : Le locuteur se réfère à l'équivalent du mot « la drogue » en arabe. Il s'agit du mot .qui est un mot masculin [moxadir] "المخدر"

•[ososijete] : Une référence au mot masculin arabe "مجخدع [modztamaç] équivalent du mot féminin français « société » est à l'origine de cette erreur.

- [ləkup] : Cette erreur est due à une interférence grammaticale où le jeune enregistré a fait référence au mot "الكاس" [alka?s]. Bien qu'il soit féminin dans la langue arabe, ce mot a un usage masculin dans l'arabe dialectal. Et c'est cela qui explique cette erreur.

-[lekotdivwaR]: L'usage de l'article masculin est dû à une interférence grammaticale où l'enquêté utilise l'équivalent de "la côte d'ivoire » en arabe. Il s'agit de "ساحل العاج " [saHilalça3] qui est un mot masculin.

-[ləpદRsõ] : Est à son tour une erreur interférentielle où le locuteur se réfère à l'équivalent masculin arabe de "la personne ». Il est question du mot "شخص 》[ [Saxs].

-[ləmєR] : Le jeune interrogé utilise l'article masculin en faisant référence au mot masculin . "équivalent arabe du mot français « la mer [baHr] «بحر》

•[ləfyme] : Est utilisé au masculin suite à une interférence au mot arabe masculin "الدخان" [adoxan] équivalent du mot français « la fumée ». Dans [lafymileemoRtEl], on remarque que

TIPA. Travaux interdisciplinaires sur la parole et le langage, 31 | 2015 
même après la correction le jeune introduit un pronom qui indique le masculin et qui renvoie à ce même mot.

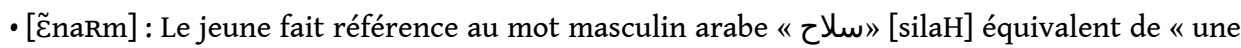
arme ».

•[mõtct] : Le mot « tête » en arabe est masculin (« راس" [raPs]). Ce qui justifie l'utilisation de «mon».

\subsubsection{La négation}

51 La négation est d'un point de vue grammatical un point de langue qui recouvre des dimensions lexico-sémantiques et syntaxiques. Dans leur ouvrage Grammaire méthodique $d u$ français, Riegel , Pellat et Rioul résument le fonctionnement de la négation en français qui selon eux pose deux problèmes:

D'une part, l'expression de la négation varie en fonction des niveaux de langue et de la distinction entre l'oral et l'écrit. Ainsi, on constate que le terme ne est souvent omis à l'oral (je veux pas), alors qu'il connaît un emploi original, (" explétif » [..]) dans un niveau recherché (je crains qu'elle ne parte). D’autre part, l'incidence syntaxique de la négation ne recouvre pas toujours sa portée sémantique ; autrement dit, la place des termes négatifs n'indique pas automatiquement quel segment est affecté par la négation. (Riegel, Pellat et Rioul, 1998 : 410).

L'omission de la négation est très répandue dans notre corpus, à titre d'exemple : [sivuzavepalətã], [ilkonepa],...Etant attestée en français oral spontané, nous ne pouvons pas la considérer comme une erreur. C'est, cependant, son usage dans l'énoncé suivant qui est pris pour une interférence : «Le temps est couteau quand ne pas coupe il te coupe $»^{3}$. La structure de la négation dans cet énoncé est semblable à celle utilisée dans la phrase arabe où la négation précède le verbe. En arabe, bien qu'elle s'effectue différemment selon le type de la phrase, selon l'aspect du verbe et le temps exprimé, la particule de la négation précède toujours le verbe.

\subsubsection{L'emploi de la préposition comme source d'interférence}

53 Une des erreurs interférentielles que nous avons pu relever de notre corpus et qui constituent selon nos enquêtés une des caractéristiques du "français cassé", est l'emploi erroné de la préposition. Notre corpus d'enregistrements montre qu'une interférence avec le système prépositionnel arabe est à l'origine de ces erreurs et donc de cette particularité.

La préposition est "une partie du discours invariable qui appartient à la catégorie des mots de relation [...]. Elle sert à relier des termes pour les intégrer dans une construction plus vaste.» (Riegel, Pellat et Rioul, 1998 : 369). Elle introduit un élément devant lequel elle se place d'où son nom de pré-position. Elle forme avec son régime une unité syntaxique et sémantique. Il s'agit d'un terme subordonnant qui contribue à la construction et aussi à l'interprétation du sens de la construction qu'il introduit. Les auteurs précédemment cités expliquent dans ce sens: "Qu'elles soient ou non porteuses d'un sens identifiable à travers la diversité de leurs emplois, les prépositions contribuent à l'établissement de relations sémantiques entre les termes qu'elles relient » (1998: 369). 

prépositionnel) est relative à la relation qu'il entretient avec les autres constituants de la phrase dans laquelle il figure (complément d'objet indirect, complément circonstanciel de temps, de lieu, complément de nom, etc.).

préposition a en effet un sens très divers selon le complément qu'elle introduit et le verbe dont elle dépend. Il existe des prépositions qui sont attachées à un des éléments qu'elles relient tels que se souvenir de, donner à, penser à, sur l'honneur, etc. C'est ainsi que certaines prépositions ont une relation avec la nature et le choix du verbe, avec le nom et la relation qu'il engage avec elles, etc.

que certaines erreurs relatives à l'usage des prépositions ont une relation avec la nature du verbe et son équivalent en arabe. C'est le cas de :

- [3ədonõ/kõscjœ/siRtu/siRtu/siRtupuRlezõfã] : L'usage de la préposition «pour» à la place de la préposition «à » est dû à une contrainte morphosyntaxique par analogie à la structure de la langue arabe. Il s'agit d'une interférence prépositionnelle en relation avec la nature de l'équivalent du verbe "donner» en arabe. En français ce verbe doit être accompagné de la préposition "à » ("donner quelque chose à quelqu'un »). Le recours au sémantisme de l'équivalent de "donner à» en arabe ("J J J... " [aÇt'ali]) traduit littéralement par « donner pour » est à l'origine de cette interférence.

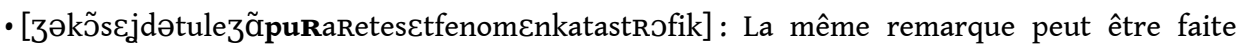
dans les propos de ce jeune. Dans cet énoncé, il convient d'utiliser la préposition «de» (" je conseille les gens d'arrêter ce phénomène »). L'utilisation de la préposition «pour » au lieu de «de » est relative à des contraintes syntaxico-sémantiques liées à la nature du verbe "conseiller " en arabe mais aussi en français. Dans cette phrase le conseil est donné dans le but d'arrêter de fumer ce qui explique l'usage de préposition «pour » équivalent de " $J$ » [li] en arabe utilisée la plupart du temps pour exprimer le but.

- [3əveparlesyRlətaba] : La confusion entre la préposition «sur » et « de » («parler sur quelque chose» au lieu de «parler de quelque chose» s'explique par une interférence avec l'arabe dialectal. Ce locuteur a pensé directement dans sa langue maternelle et a produit l'équivalent français de la phrase [natkalmçala] ([naHdarçla]) où "على " [Çala] est synonyme de «sur».

Notre corpus a permis, en outre, de dévoiler certaines erreurs interférentielles en relation avec les prépositions spatiales. Les différences entre les systèmes prépositionnels arabe et français en ce qui concerne les prépositions topologiques entraînent un certain nombre d'erreurs qui constituent, selon nos enquêtés, une des particularités du "français cassé ». Utiliser une préposition à la place d'une autre est dû à des contraintes syntaxiques par analogie au système de l'arabe. Les prépositions spatiales « $\grave{a}$ ", « dans », «en », par exemple, ont, en français, un usage particulier selon des aspects sémantiques et morpho-syntaxiques. En arabe, ces trois prépositions ont pour équivalent la préposition "في " [fi], traduite le plus souvent par "dans" en français. Ce qui apparait dans les exemples suivants :

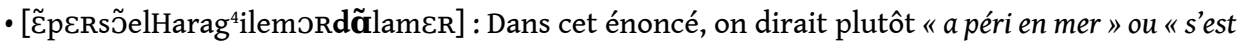
noyé». L'usage de «dans» dans ce contexte est dû à une interférence avec le système de la langue arabe où le locuteur traduit littéralement [filbaHr] :

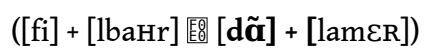

-[ləRəkẼasidãlatabldəmãdləməny]: L'utilisation de la préposition «dans» dans cette production constitue une interférence avec le système de l'arabe. Cette préposition comme 
nous l'avons mentionné précédemment envisage le lieu comme un volume et non comme un point topographique statique. Or, il convient d'utiliser la préposition «à»: [ləRəkẼasiatabldəmõdləməny] («à table »). Cette erreur interférentielle s'explique par une transposition du sémantisme de l'arabe dialectal [m3amaçfitabla] où la préposition [fi] équivaut à (" في (") «ans ».

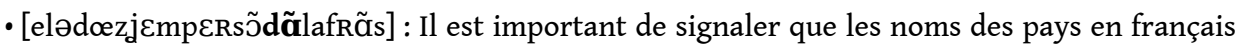
se forment en utilisant la préposition «à " quand le nom est masculin à initiale consonantique. Quand le nom du pays est féminin ou quand il commence par une voyelle on utilise la préposition «en». Ce qui est le cas dans cette production. Le mot «France» est féminin. Il convient donc d'utiliser la préposition «en». L'usage erroné de la préposition «à » constitue dans cet énoncé une erreur interférentielle. Le locuteur fait appel à la structure de l'arabe et utilise « dans » qui traduit le plus souvent [fi] («ي ").

\subsubsection{L'emploi de l'article contracté}

\section{enregistrés utilisent séparément la préposition et l'article dans leurs productions en français au lieu de les amalgamer. Cela est expliqué par une interférence avec le système de l'arabe. C'est le cas de :}

•[ijadezadolsãkisavpa....la.....kisavpalekõsəkõsdələtaba] : au lieu de [dytaba]

En traduisant l'expression arabe : "نتائج التدخين " [nataizatadxin], ce jeune n'a pas su utiliser l'article contracté « $d u »$ puisque, comme nous l'avons cité plus haut, ce dernier n'existe pas en arabe.

-[õvwamemlefamile../ilepartiavek/alebatodeHaraga] : au lieu de [obato]

L'usage de «à»+ «les» dans cet énoncé est le résultat d'une interférence avec la forme arabe " في الباخرات " [fialbaxirat]. Une traduction mot à mot de cet énoncé a entraîné l'usage des deux prépositions.

$61[\mathrm{fi}]+[\mathrm{al}]+[$ baxira $]$ 臨 $[\mathrm{a}]+[\mathrm{le}]+[$ bato $]$

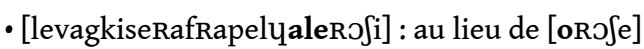

Il s'agit d'une transposition de la forme arabe " في الصخرة : [fiasoxur] au français :

\subsubsection{L'emploi du pronom personnel complément}

L'usage erroné des pronoms personnels en français et surtout des pronoms personnels compléments est l'une des erreurs qu'on a pu relever de notre corpus. Ce genre d'imperfections interférentielles constitue une des caractéristiques du français dit « cassé ».

TIPA. Travaux interdisciplinaires sur la parole et le langage, 31 | 2015 

devant le verbe aux temps simples et composés. Dans le cas de la négation, ils se placent entre la première négation et le verbe. Leur position est donc toujours avant le verbe. En arabe, ils ont la même fonction sauf qu'ils sont toujours suffixés au verbe et ils se placent après ce dernier.

Une interférence des deux systèmes arabe et français fait que les locuteurs dont les propos sont les suivants placent les pronoms après les verbes :

- [levagkiserafRapelyaleRJSi] : Ce jeune utilise le pronom personnel «lui» (au lieu de «le»qui le frappe-) après le verbe. Cet usage est dû non seulement à une non maîtrise du système des pronoms personnels compléments en français mais aussi à une interférence avec le système de l'arabe qui est considéré comme plus complexe en ce qui concerne cette question.

-[səsว̃dezimazkiõtufemwa] : Ce jeune utilise aussi le pronom «moi» à la place du pronom personnel complément d'objet direct «me »-qui m'ont touché-). L'usage de ce pronom après le verbe constitue une interférence avec le système de l'arabe.

\subsubsection{L'emploi du pronom relatif}

Les divergences entre le système des relatives arabes et françaises entrânent un certain nombre d'interférences et c'est ce qu'on a pu observer dans notre corpus. En français, le pronom relatif introduit une proposition subordonnée et évite la répétition. La même fonction est attribuée à cet élément dans la relative arabe ${ }^{5}$.

Selon Grevisse et Goosse, les pronoms relatifs :

[appelés aussi conjonctifs] servent à introduire une proposition, qu'on appelle ellemême relative ; à la différence des conjonctions de subordination (qui introduisent aussi une proposition, ils ont une fonction dans cette proposition : celle de sujet, de complément, parfois d'attribut; ils ont un genre, un nombre, une personne, même s'ils n'en portent pas visiblement les marques (...) s'ils sont représentants, ils ont un antécédent. (2008: 909)

La particularité du français en ce qui concerne l'emploi des pronoms relatifs réside donc dans le fait que la forme de ceux-ci dépend de leur fonction (qui : sujet, que : COD, etc.).

70 A l'encontre du pronom relatif français, la forme de celui de l'arabe est indépendante de sa fonction. Selon Ben Amor Ben Hamida la morphosyntaxe du pronom relatif arabe est différente dans le mesure où "il n'y a pas de changement de la forme de base, qu'il

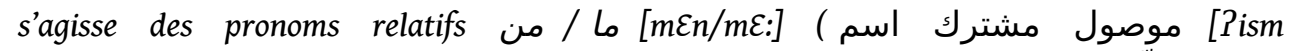
mawsu:lmuftErEk] (pronom relatif qui reprend un humain/ un non humain) ou الّّ [? [? [?ismmawsu:l XE:s]. » (موصول خاص/سم :113).

71 Ainsi, «que » qui remplace un COD, «qui » qui remplace un sujet et « où » (complément circonstanciel de lieu ou de temps) ${ }^{6}$ ont un seul équivalent en arabe qui est le pronom relatif "الذي " [alaði]” (tout en l'accordant en genre et en nombre avec son antécédent). 
C'est cela qui explique l'emploi du pronom relatif «qui » au lieu de «que» dans les propos relevés dans notre corpus :

-[lẽmportãskiazuwesəmat [] : Il s'agit ici d'une erreur interférentielle commise par un des jeunes interrogés. C'est une confusion qui trouve son origine dans la particularité morphosyntaxique du pronom relatif arabe.

\subsubsection{La forme pronominale des verbes}

Nous avons pu dévoiler à travers l'analyse linguistique de notre corpus des enregistrements, des erreurs interférentielles en relation avec la forme pronominale des verbes. Les locuteurs interrogés dans ce cas se réfèrent soit à l'arabe standard soit à l'arabe dialectal lors de leurs productions en langue française.

Dans l'énoncé [3ez̃skRio../obakpuR/puRganjeləbakynotRfwa], le jeune interrogé fait appel à l'arabe dans ses deux formes et c'est cela qui constitue la source de l'erreur commise dans l'usage de la forme pronominale du verbe " inscrire » (« je me suis inscrit » et non «j'ai inscrit »). Il s'agit d'une traduction de la construction [sazaltofilbakalorja] (en arabe standard) ou [sazaltfilbak] (ou même [ع̃skritfilbak] en arabe dialectal) dans laquelle l'accent est mis sur l'action faite par le sujet et non sur le sujet lui-même. En d'autres termes, l'acte se présente comme accompli. Ce qui justifie l'emploi de l'auxiliaire « avoir».

\section{L'interférence linguistique dans l'usage du « français cassé » : paramètres socioculturels}

Etudier les interférences dans les pratiques linguistiques des bilingues, c'est donc interpeler des éléments linguistiques (qui se rapportent à la structure de la langue source et à celle de la langue cible), des éléments socioculturels et psychologiques de l'individu bilingue mais aussi des éléments situationnels (dépendants de paramètres liés à la situation de communication tels que le milieu, le registre et le style qui en dépendent, le thème, etc.). Le nombre des interférences et leur nature sont étroitement liés à l'histoire socio-langagière des locuteurs dans la langue source et dans la langue cible.

Nous nous attacherons dans cette étape de l'étude à analyser et à interpréter le recours à l'interférence dans les productions en « français cassé » en prenant en considération les facteurs extralinguistiques.

Dans le but d'avoir une vue d'ensemble sur la répartition des interférences selon les paramètres socio-culturels (sexe, âge et niveau d'étude), nous établissons les tableaux ${ }^{8}$ ci-dessous. Les interférences sont réparties selon la durée de l'enregistrement représentée dans les tableaux par la lettre « $\mathrm{D}^{9} »$.

\begin{tabular}{|l|l|l|l|l|l|l|}
\hline \multicolumn{2}{|l|}{ D1 } & \\
\hline \multirow{2}{*}{ Sexe } & Niveau d'étude & Moins de 20 & $\mathbf{2 0 - 2 4}$ & $\mathbf{2 5 - 2 9}$ & $\mathbf{3 0}$ et plus & Total \\
\hline \multirow{2}{*}{ Femme } & Universitaire & $1(1)$ & $3(9)$ & $2(4)$ & & $\mathbf{6}(\mathbf{1 4})$ \\
\cline { 2 - 7 } & Terminale & $1(1)$ & $1(1)$ & $1(3)$ & & $\mathbf{3}(5)$ \\
\hline
\end{tabular}




\begin{tabular}{|l|l|l|l|l|l|l|}
\hline \multirow{2}{*}{ Homme } & Universitaire & & $2(4)$ & $1(2)$ & & $3(6)$ \\
\cline { 2 - 7 } & Terminale & $1(1)$ & $1(2)$ & & $1(3)$ & $\mathbf{3}(6)$ \\
\hline \multirow{2}{*}{ Total } & $3(3)$ & $7(16)$ & $\mathbf{4 ( 9 )}$ & $\mathbf{1 ( 3 )}$ & $15(31)$ \\
\hline
\end{tabular}

TABLEAU N N ${ }^{\circ}$ : Classification des interférences relevées dans le corpus d'enregistrement en D1.

\begin{tabular}{|l|l|l|l|l|l|l|}
\hline \multicolumn{2}{|l|}{ D2 } \\
\hline \multirow{2}{*}{ Sexe } & Niveau d'étude & Moins de 20 & $\mathbf{2 0 - 2 4}$ & $\mathbf{2 5 - 2 9}$ & $\mathbf{3 0}$ et plus & Total \\
\hline \multirow{2}{*}{ Femme } & Universitaire & & & & $1(8)$ & $\mathbf{1}(\mathbf{8})$ \\
\cline { 2 - 7 } & Terminale & & & & & \\
\hline \multirow{2}{*}{ Homme } & Universitaire & & & $1(15)$ & $1(10)$ & $\mathbf{2}(\mathbf{2 5})$ \\
\cline { 2 - 7 } & Terminale & & $1(17)$ & & & $\mathbf{1}(\mathbf{1 7})$ \\
\hline \multirow{2}{*}{ Total } & & & $\mathbf{1}(\mathbf{1 7})$ & $\mathbf{1 ~ ( 1 5 )}$ & $\mathbf{2}(\mathbf{1 8})$ & $4(50)$ \\
\hline
\end{tabular}

TABLEAU N8 : Classification des interférences relevées dans le corpus d’enregistrement en D2.

\begin{tabular}{|l|l|l|l|l|l|l|}
\hline \multicolumn{2}{|l|}{ D3 } \\
\hline \multirow{2}{*}{ Sexe } & Niveau d'étude & Moins de 20 & $\mathbf{2 0 - 2 4}$ & $\mathbf{2 5 - 2 9}$ & $\mathbf{3 0}$ et plus & Total \\
\hline \multirow{2}{*}{ Femme } & Universitaire & & & & & \\
\cline { 2 - 7 } & Terminale & & & & & \\
\hline \multirow{2}{*}{ Homme } & Universitaire & & & $1(24)$ & & $\mathbf{1}(\mathbf{2 4})$ \\
\cline { 2 - 7 } & Terminale & & & & & \\
\hline \multirow{2}{*}{ Total } & & & & $\mathbf{1}(\mathbf{2 4})$ & & $1(24)$ \\
\hline
\end{tabular}

TABLEAU N ${ }^{\circ}$ 9: Classification des interférences relevées dans le corpus d'enregistrement en D3.

Les résultats présentés dans les tableaux $n^{\circ} 7,8$ et 9 montrent que :

- La majorité des sujets sont classés en « D1 » (soit 15/20).

- La majorité des jeunes enregistrés du niveau «terminale » (soit 6/7) sont classés en «D1 ». Cette durée est marquée par un nombre d'interférences variant entre 1 et 4 .

- Les locutrices ont produit entre 1 à 8 interférences. La moyenne des interférences chez les locuteurs de sexe masculin est entre 1 et 24 interférences.

- La majorité des interférences sont produites par les locuteurs du niveau universitaire.

- Plus les locuteurs enregistrés sont âgés, plus ils produisent des interférences. Cela est déduit à travers la lecture de chacun des trois tableaux. L'usage des interférences semble très 
largement caractéristique des jeunes entre 20 et 29 ans. En effet, 81 des 105 interférences relevées du corpus ont été produites par les jeunes de cette tranche d'âge.

Il ressort, d'une façon générale, de cette analyse que les paramètres de sexe, de l'âge, du niveau d'étude et de la durée se sont révélés significatifs dans la production des interférences dans l'usage du «français cassé ».

- Le sexe

Bien que la majorité des locutrices aient été enregistrées en «D1", elles produisent moins d'interférences que les hommes. Cela est expliqué par le fait que les femmes font plus attention quand elles parlent. Les études sociolinguistiques laboviennes montrent que les femmes sont beaucoup plus disposées à l'adoption d'une langue standard et à la conformité linguistique. Pillon affirme pour sa part que «les femmes, ont tendance, dans les situations formelles et, donc, aussi sans doute les situations expérimentales à modifier leurs pratiques linguistiques en fonction des attentes dominantes » (1987:14). Les femmes veillent donc à produire des énoncés corrects et insistent sur la bonne prononciation. Lakoff (1973) attribue d'ailleurs aux femmes la qualité de «pruderie linguistique ».

- L'âge et le niveau d'étude

80 Nous avons choisi de réunir ces deux variables parce que nous considérons que les résultats à leur propos sont liés. A propos de l'influence de l'âge et du niveau d'étude sur le recours à la première langue dans l'usage $d u$ " français cassé », l'analyse montre que les jeunes de plus de 20 ans et surtout ceux du niveau universitaire commettent plus d'interférences dans leurs productions en «français cassé » que ceux des autres tranches d'âge et du niveau «terminale ». Il est vrai que le nombre des locuteurs n'est pas équitable entre les différentes catégories dans notre corpus mais l'observation des nombres des interférences chez chaque locuteur nous guide vers cette déduction. Les résultats à propos de ces deux variables sont un peu surprenants parce que plus le locuteur est âgé plus sa maîtrise des langues est bonne mais cela dépend de plusieurs facteurs qui touchent au degré du bilinguisme de l'enquêté.

81 Le locuteur algérien vit dans un climat plurilingue et devient bilingue à travers le temps. Cela dépend de plusieurs facteurs. Le degré du bilinguisme dépend de la durée du contact avec la deuxième langue. Qu'elle soit une langue enseignée, une langue d'enseignement ou une langue d'usage quotidien, cela influe son usage. Selon Mackey, " une langue enseignée à l'école comme matière fournira vraisemblablement moins d'heures de contact que la langue utilisée comme instrument d'enseignement » (1976 : 387).

La fréquence d'emploi de cette langue joue, selon le même auteur, un rôle important dans la délimitation du degré du bilinguisme du locuteur. Il est question ici de savoir si l'usager utilise fréquemment la deuxième langue ou bien dans quelques situations seulement. Mackey (1976) note également que le degré du bilinguisme est tributaire de la «pression». Il s'agit du contexte d'usage de la langue seconde et des forces qui poussent le locuteur à l'utiliser. Il peut s'agir de forces économiques, historiques, sociales, administratives, etc.

83 Enfin, «l'attitude du locuteur est un facteur important de la description de son bilinguisme» (Mackey, 1976: 394). La représentation du locuteur envers la langue et ses locuteurs influencera son comportement langagier; soit lors de l'usage de la langue ou même dans les situations dans lesquelles cette langue est utilisée (quand elle est valorisée ou dévalorisée au sein de la société ou même parmi les membres du groupe d'appartenance). 


\section{communication, le jeune est confronté à des réalités et des situations nouvelles dans} lesquelles il cherche à développer sa bilingualité ${ }^{11}$ à travers l'usage du français. Les erreurs commises dans nos enregistrements définissent une compétence en cours de développent. C'est ainsi que le français produit par ces jeunes, français qu'ils considèrent comme « cassé » reflète un effort d'apprentissage et un souci de bien parler cette langue.

\section{Conclusion}

Au cours de ce travail, nous avons voulu nous investir dans un domaine qui relie le fait linguistique au sociolinguistique. Il s'agit d'une étude sur les manifestations du " français cassé » utilisé en Algérie. Cet usage résulte du contact entre le français et l'arabe (avec ses variétés). C'est une réalisation particulière de la langue française liée, nous semble-t-il, à l'ensemble des images et des représentations de cette langue et à la dynamique des systèmes linguistiques en présence, dans divers contextes. Cet usage constitue aussi une particularité du français parlé en Algérie. 
89 en quoi l'usage du "français cassé » s'écartait de l'usage du français de référence. Le traitement de données a été fait par le biais d'une analyse contrastive des différentes productions constituant notre corpus de recherche.

L'analyse des données a permis de montrer que l'interférence est à la base de ce que les jeunes algériens appellent le «français cassé ». Tout en utilisant le français, le locuteur algérien se réfère à la structure et au fonctionnement (phonétique, lexical, syntaxique et sémantique) de sa langue, dont les structures sont incompatibles avec celles du français. Les facteurs socio-culturels sont certes révélateurs dans la production des interférences mais il ne faut pas négliger le rôle de l'implication représentationnelle du locuteur qui pourrait aussi être à l'origine des interférences, lors de l'usage du français ce qui le rend dans notre cas : « cassé ».

\section{BIBLIOGRAPHIE}

Bahloul N. (2009) L'arabe dialectal, un outil pour une intercompréhension en classe de langue, Synergie Algérie, $\mathrm{n}^{\circ}$ 4, p. 255-263.

Ben Amor Ben Hamida T. (2009) Erreurs interférentielles arabe-français et enseignement du français, Synergie Tunisie, $\mathrm{n}^{\circ} 1$, p. 105-117.

Derradji Y. (1999) Le français en Algérie : langue emprunteuse et empruntée, français en Afrique, revue du Réseau des observatoires du français contemporain en Afrique, p.71-82. Disponible sur : http://www.unice.fr/ILF-CNRS/ofcaf/13/derradji.html\#1 consulté le 10/11/2012

Fesfes N. (1994) Problèmes de l'apprentissage du français langue étrangère par des élèves syriens de l'enseignement secondaire, Université de Toulouse.

Grevisse, M. (1993) Le bon usage : grammaire française, refondue par Goosse A., $13^{\mathrm{e}}$ Paris, édition revue, Duculot. (14e édition revue, De Boeck et Larcier, 2008).

Hagege C. (1996) L'enfant aux deux langues, Paris : Odile Jacob.

Hamers J. \& Blanc J- M. (1983) Bilingualité et bilinguisme, Bruxelles : Margada.

Hassan A. (1974) Interférence, linguistique contrastive et sa relation avec l'analyse des fautes, $\mathrm{La}$ pédagogie des langues vivantes, $\mathrm{n}^{\circ} 5$. p. 168-173.

Kannas C. (1994) Dictionnaire de linguistique et des sciences du langage, Paris : Larousse.

Lakoff R. (1973) Language and women, Language in Society, vol. 2, n 1 , Cambridge : JSTOR, p. 45-80.

Mackey W. (1976) Bilinguisme et contact de langues, Paris : Klincksieck.

Maume J.-L. (1973) L'apprentissage du français chez les arabophones maghrébins (diglossie et plurilinguisme en Tunisie), Langue française, $\mathrm{n}^{\circ} 19$, p. 90-107.

Medane H. (2014) Le « français cassé » chez les jeunes Algériens : Interférence et calque au service de l'humour, actes du CMLF 2014- 4ème Congrès Mondial de Linguistique Française, p. 1669-1683, publié par EDP Sciences (www.linguistiquefrancaise.org).

TIPA. Travaux interdisciplinaires sur la parole et le langage, 31 | 2015 
Pillon A. (1987) Le sexe du locuteur est-il un facteur de variation ?, La Linguistique, vol. 23, Paris : PUF, p. 35-48

Riegel M., Pellat J-C. \& Rioul R. (1998) Grammaire méthodique du français, Paris : PUF.

Tabouret Keller, A. (2008) L'expression contact comme révélatrice de la dynamique des langues, Journal of language contact, Thema 2, p. 7-18.

Taleb-Ibrahimi K. (1997) Les Algériens et leur(s) langue(s), Alger : El Hikma.

Walter H. (1988) Le Français dans tous les sens, Paris : Laffont.

\section{ANNEXES}

1. Conventions de transcription

\begin{tabular}{|c|c|}
\hline \multicolumn{2}{|c|}{ Transcription des phonèmes de l'arabe } \\
\hline q & 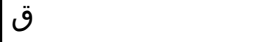 \\
\hline r & ر \\
\hline $\mathrm{x}$ & $\dot{\tau}$ \\
\hline $\mathrm{H}$ & $\tau$ \\
\hline $\mathrm{H}$ & ه \\
\hline Ç & $\varepsilon$ \\
\hline Đ & ذ \\
\hline$\partial^{\mathrm{i}}$ & 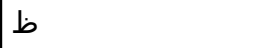 \\
\hline$t^{\mathrm{i}}$ & $b$ \\
\hline$d^{\text {द }}$ & ض \\
\hline$\Theta$ & $ث$ \\
\hline$?$ & $s$ \\
\hline
\end{tabular}

2. Profil des enquêtés

\begin{tabular}{|l|l|l|l|l|l|l|}
\hline Informateur & Sexe & Age & Fonction & Résidence & Origine & $\begin{array}{l}\text { Profession des } \\
\text { parents }\end{array}$ \\
\hline L43 (02) & Mas. & $\begin{array}{l}21 \\
\text { ans }\end{array}$ & étudiant & Chlef & Ain Defla & $\begin{array}{l}\text { Père : chômeur } \\
\text { Mère : femme } \\
\text { au foyer }\end{array}$ \\
\hline
\end{tabular}




\begin{tabular}{|c|c|c|c|c|c|c|}
\hline L22 (02) & Mas. & $\begin{array}{l}33 \\
\text { ans }\end{array}$ & coiffeur & Chlef & Chlef & $\begin{array}{l}\text { Père : paysan } \\
\text { Mère : femme } \\
\text { au foyer }\end{array}$ \\
\hline L48 (02) & Mas & 23ans & étudiant & Chlef & Chettia & $\begin{array}{l}\text { Père : taxieur } \\
\text { Mère : femme } \\
\text { au foyer }\end{array}$ \\
\hline L47 (02) & Mas & $\begin{array}{l}22 \\
\text { ans }\end{array}$ & étudiant & Chlef & Sobha & $\begin{array}{l}\text { Père : fellah } \\
\text { Mère: femme } \\
\text { au foyer }\end{array}$ \\
\hline L50 (02) & Mas & $\begin{array}{l}18 \\
\text { ans }\end{array}$ & $\begin{array}{ll}\text { ne } & \text { travaille } \\
\text { pas } & \end{array}$ & Chlef & Tachna & $\begin{array}{l}\text { Père : prof } \\
\text { Mère : femme } \\
\text { au foyer }\end{array}$ \\
\hline L45 (02) & Mas & $\begin{array}{l}22 \\
\text { ans }\end{array}$ & commerçant & Chlef & Ain Defla & $\begin{array}{l}\text { Père : retaité } \\
\text { Mère: femme } \\
\text { au foyer }\end{array}$ \\
\hline L42 (02) & Fem & $\begin{array}{l}21 \\
\text { ans }\end{array}$ & étudiante & Chlef & $\begin{array}{l}\text { Ain } \\
\text { Mérane }\end{array}$ & $\begin{array}{l}\text { Père : } \\
\text { infirmier } \\
\text { Mère : femme } \\
\text { au foyer }\end{array}$ \\
\hline L44 (02) & Mas & $\begin{array}{l}21 \\
\text { ans }\end{array}$ & électricien & Chlef & Alger & $\begin{array}{l}\text { Père : } \\
\text { surveillant } \\
\text { général au } \\
\text { lycée } \\
\text { Mère : / }\end{array}$ \\
\hline L41 (02) & Mas & $\begin{array}{l}30 \\
\text { ans }\end{array}$ & $\begin{array}{l}\text { infirmier } \\
\text { + étudiant }\end{array}$ & Chlef & Oum Drou & $\begin{array}{l}\text { Père : émigré } \\
\text { Mère : femme } \\
\text { au foyer }\end{array}$ \\
\hline L $46(02)$ & Mas & $\begin{array}{l}22 \\
\text { ans }\end{array}$ & étudiant & Chlef & $\begin{array}{l}\text { Khémis } \\
\text { Méliana }\end{array}$ & $\begin{array}{l}\text { Père : retraité } \\
\text { Mère : / }\end{array}$ \\
\hline L $40(02)$ & Fem & $\begin{array}{l}29 \\
\text { ans }\end{array}$ & fonctionnaire & Chlef & El Attaf & $\begin{array}{l}\text { Père : retraité } \\
\text { Mère : } \\
\text { enseignante }\end{array}$ \\
\hline L39 (02) & Fem & $\begin{array}{l}22 \\
\text { ans }\end{array}$ & étudiante & Chlef & Boukadir & $\begin{array}{l}\text { Père : émigré } \\
\text { Mère : retraité }\end{array}$ \\
\hline
\end{tabular}




\begin{tabular}{|c|c|c|c|c|c|c|}
\hline L38 (02) & Mas & $\begin{array}{l}29 \\
\text { ans }\end{array}$ & $\begin{array}{l}\text { vendeur en } \\
\text { pharmacie }\end{array}$ & Chlef & Elabadia & $\begin{array}{l}\text { Père : décédé } \\
\text { Mère : femme } \\
\text { au foyer }\end{array}$ \\
\hline L49 (02) & Mas & $\begin{array}{l}25 \\
\text { ans }\end{array}$ & prof. de dessin & Chlef & $\begin{array}{l}\text { Oued el } \\
\text { Fedda }\end{array}$ & $\begin{array}{l}\text { Père : retraité } \\
\text { Mère: femme } \\
\text { au foyer }\end{array}$ \\
\hline L 54 (13) & Fém. & $\begin{array}{l}19 \\
\text { ans }\end{array}$ & étudiante & Tlemcen & Sebdou & $\begin{array}{l}\text { Père : policier } \\
\text { Mère : femme } \\
\text { au foyer }\end{array}$ \\
\hline L 55 (13) & Fém & $\begin{array}{l}26 \\
\text { ans }\end{array}$ & $\begin{array}{ll}\text { ne } & \text { travaille } \\
\text { pas } & \end{array}$ & Tlemcen & Chouli & $\begin{array}{l}\text { Père : } \\
\text { fonctionnaire } \\
\text { Mère : femme } \\
\text { au foyer }\end{array}$ \\
\hline L53 (13) & Mas & $\begin{array}{l}18 \\
\text { ans }\end{array}$ & élève & Tlemcen & Nédroma & $\begin{array}{l}\text { Père : ancien } \\
\text { directeur de } \\
\text { CEM } \\
\text { Mère: femme } \\
\text { au foyer }\end{array}$ \\
\hline L56 (13) & Mas & $\begin{array}{l}28 \\
\text { ans }\end{array}$ & chômeur & Tlemcen & Oran & $\begin{array}{l}\text { Père : retraité } \\
\text { Mère : / }\end{array}$ \\
\hline L83 (13) & Mas & $\begin{array}{l}23 \\
\text { ans }\end{array}$ & commerçant & Tlemcen & Sabra & $\begin{array}{l}\text { Père : médecin } \\
\text { Mère } \\
\text { enseignante }\end{array}$ \\
\hline L 84 (13) & Fém & $\begin{array}{l}30 \\
\text { ans }\end{array}$ & fonctionnaire & Tlemcen & $\begin{array}{l}\text { Béni } \\
\text { Snous }\end{array}$ & $\begin{array}{l}\text { Père : } \\
\text { boulanger } \\
\text { Mère } \\
\text { couturière }\end{array}$ \\
\hline
\end{tabular}

\section{NOTES}

1. Nous avons proposé six thèmes : le tabac, la violence scolaire et le système éducatif, les voyage clandestins, le match Algérie/Côte d'Ivoire et la sécurité routière.

2. Girro: i girro (cigarette); pl. i girroten, igirowan.

3. Il s'agit d'un proverbe arabe traduit littéralement de l'arabe standard au français. La structure de l'unité phraséologique arabe [əlwaqtosajfoninlamtaqtaçhoqataçak] a pour équivalent en français : «Le temps est précieux ».

4. [Harag] est un terme de l'arabe dialectal désignant le voyageur clandestin. 
5. Le pronom relatif introduit une proposition relative qui doit contenir un pronom de rappel (visible ou caché).

6. Le pronom relatif « dont » n'existe pas en arabe.

7. Ou " من " $" \mathrm{men}]$ l'invariable

8. Dans ces tableaux, nous retrouvons les facteurs extralinguistiques liés aux locuteurs enregistrés. Il s'agit de l'âge du locuteur (réparti selon quatre tranches d'âge), du sexe et du niveau d'étude. Dans chaque catégorie de ces tableaux, il existe des cellules qui contiennent le nombre des locuteurs suivi du nombre des interférences mis entre parenthèses.

9. D1 : entre $00: 50$ et $01: 59 \mathrm{mn}$; D2 : entre $02: 00$ et $3: 59 \mathrm{mn} ; \mathrm{D} 3: 04: 00 \mathrm{mn}$ et plus.

Il est à noter que notre répartition des locuteurs et des interférences apparues dans leur usage du « français cassé » selon trois durées d'enregistrement est dû à un souci interprétatif des résultats obtenus. Logiquement un locuteur enregistré en «D1» commet moins d'erreurs qu'un autre enregistré sur une durée plus longue («D2» ou «D3»). Cette répartition n’a pas été faite selon les compétences déclarées par ces jeunes.

10. La compétence dans la langue maternelle est supérieure à celle en français.

11. Il existe bien sûr plusieurs moyens qui peuvent aider un locuteur pour améliorer son niveau en français : lire, regarder des émissions en français,...

\section{RÉSUMÉS}

Notre étude s'inscrit dans un domaine qui relie le fait linguistique au sociolinguistique. Elle vise la description et l'analyse des manifestations du «français cassé ». A travers l'analyse des productions de 20 jeunes algériens et en nous basant sur le principe de l'analyse contrastive, nous allons voir comment l'interférence constitue une des particularités de cet usage.

In Algeria, linguistic diversity has been the subject of several studies. The resulting sociolinguistic facts identify the degree of contact and mixing of languages in Algeria. The languages presented in this area, and which coexist with each other, do not share the same importance or the same prestige. The coexistence of two or more languages implies that they are used in different contexts, regions and even interlocutors, by members of the company to fulfill various functions.

The Algerian speaker uses all the available language resources in his linguistic landscape, which has fostered the creativity of the subject speaking Algerian in French. The language practices of French by Algerian speakers are constantly changing. This innovation "is distinguished in its form and content in a clear difference from the exogenous norm of the standard French." (Derradji, 1999:71).

Based on the observation of the complexity related to the coexistence of languages in Algeria, the symbolic values that these languages convey and the particular use of French in Algeria, we are interested in what Algerians call the "broken French". It is a phenomenon resulting from contacts between the French and the languages or language varieties, including their performances, which are presented in the Algerian linguistic landscape. This is an incorrect implementation of the French language related, it seems, to all the images and representations of the language. Therefore, it is about a particular use of French by Algerian speakers. This linguistic practice forms the peculiarity of the spoken French in Algeria. It is about the use of the 
French language in which many phenomena are present: interference, calque, neologisms, borrowing...etc.

During this work, we wanted to investigate in an area that connects the linguistic fact to sociolinguistics. This is a study of the manifestations of the "broken French" used in Algeria. This usage results from contact between the French and Arabic (with its varieties). This is an incorrect implementation of the French language related, it seem, to all the images and representations of the language and dynamics of linguistic systems that are present in various contexts. This usage is also a feature of spoken French in Algeria.

Our contribution is a part of an investigation of the peculiarities of the "broken French" used in Algeria. Our work is the description and analysis of the manifestations of this phenomenon. We offer a qualitative approach through the analysis of 20 young Algerians productions. Based on the principle of contrastive analysis, we will see how the interference is one of the peculiarities of this use. This study attempts also to see if the linguistic interferences constituting the use of French called "broken" are related to extra-linguistic factors and / or specific representations.

Our research was conducted in two Algerian cities: Chlef and Tlemcen. We asked 20 young people to comment on a number of images that deal with topics related to Algerian youth using what they call the "broken French", using recording process. Depending on this technique of investigation, we aim an analysis of language data while observing the peculiarities of the phenomenon of "broken French".

After listening to the recordings of the young respondents, we sought the mistakes made by young people. In order to group them into different types, we performed several plays. First, we tried to identify then we classified them for interpretation. The constitution and the observation of the corpus were possible because of the transcription of recorded productions.

After the analysis of errors committed by youth during the recordings, it appears that the incorrect use of French in what they call the "broken French" is due to the phenomenon of interference. In this paper, we do not report all kinds of errors but only those involving the interference of the two languages: Arabic and French. The errors revealed by the corpus allowed us to distinguish three types of interference: phonetic, lexical-semantic and syntactic.

Data analysis has shown that interference is the basis of what the Algerian young people call the "broken French". While using the French, Algerian speaker refers to the structure and function (phonetic, lexical, syntactic and semantic) of his language, that its structures are incompatible with that of the French. Thus, the socio-cultural factors are certainly indicative in the production of interference but do not forget the role of representational implication of the speaker, which could also cause interference during the use of French making our case: "broken".

\section{INDEX}

Mots-clés : Français en Algérie, français cassé, interférence, pratiques langagières, sociolinguistique

Keywords : French in Algeria, broken French, interference, language practices, sociolinguistics

\section{AUTEUR}

\section{HADJIRA MEDANE}

Université Hassiba Ben Bouali-CHLEF (Algérie)

Laboratoire Dylandimed (Tlemcen)

medane_hadjira@yahoo.fr 